\title{
Search for ultra-high energy neutrinos from below and above the horizon with the ANTARES Telescope
}

\section{Chiara Perrina ${ }^{* \dagger}$}

"La Sapienza" University and INFN, Roma, Italy

E-mail: chiara.perrina@romal.infn.it

\begin{abstract}
Over one century has passed since the discovery of cosmic rays and their origin is still uncertain. The multi-messenger approach seems to be the key in this area of research. Neutrinos are among the best cosmic messengers thanks to the fact they are neutral, stable and weakly interacting. ANTARES is the first undersea Neutrino Telescope, its main purpose is the search for neutrino fluxes from astrophysical objects. It is placed on the bottom of the Mediterranean Sea, $\sim 40 \mathrm{~km}$ south-east from the coast of Toulon (France). Its observation is based on the detection of the Cherenkov radiation induced by the passage in water of superluminal charged particles produced by the interaction of cosmic neutrinos near the detector. ANTARES is taking data since 2007, until now we have analyzed the data collected up to 2012. No neutrino signals have been found above the expected background and upper limits on the flux normalization of an $E_{v}^{-2}$ energy spectrum have been set. In order to limit the background from down-going atmospheric muons, the analysis strategy focuses on the so-called "up-going" events, which are those that have traversed the Earth. The development of a strategy for the reconstruction and the identification of "downgoing" neutrinos is in progress. By including them in the analysis it will be possible to enlarge the field of view of ANTARES and the accessible energy range up to the EeV.
\end{abstract}

XVI International Workshop on Neutrino Telescopes,

2-6 March 2015

Palazzo Franchetti - Istituto Veneto, Venice, Italy

${ }^{*}$ Speaker.

†n behalf of the ANTARES Collaboration. 


\section{The ANTARES detector}

ANTARES (Astronomy with a Neutrino Telescope and Abyss environmental RESearch) is the first undersea Neutrino Telescope and the only one currently operating [1]. It is located at $\sim 2475$ $\mathrm{m}$ deep in the Mediterranean Sea, $\sim 40 \mathrm{~km}$ south-east off the coast from Toulon (France). The detector is composed by twelve 450-metres high strings. Each string accommodates 25 storeys with 3 photomultiplier tubes (PMTs). Acoustic devices and inclinometers regularly spread along the strings and allow to accurately monitor the position and orientation of the PMTs [2]. The detector time calibration is performed by means of an in situ array of laser and LED beacons [3]. The PMTs are orientated at $45^{\circ}$ downwards in order to maximize the sensitivity to Cherenkov light from up-going muons. The median angular resolution achieved for muon tracks assuming an energy spectrum $\propto E_{v}^{-2}$ is $<0.4^{\circ}$ allowing good performance in the searches for neutrino point sources. Its location in the Northern Hemisphere allows for surveying most of the Galactic Plane, including the Galactic Centre, thus complementing the sky coverage of the IceCube detector installed at the South Pole.

\section{Search for cosmic neutrinos from below the horizon}

The search for cosmic neutrinos from a point-like source consists in the search for a directional clustering of events. A source can be identified as a significant excess of tracks from a given location in the sky compared to the surrounding region dominated by the isotropic atmospheric background. This search can be performed either blindly over the full sky, or in the direction of $a$ priori list of candidate source locations that correspond e.g. to known gamma-ray emitters. The analysis presented here used ANTARES up-going data collected between February 2007 and December 2012, for a total live-time of 1338 days ([4]). The final neutrino sample has been obtained after tight cuts on the reconstruction quality parameter $(\Lambda)$, on the estimated angular resolution $\left(<1^{\circ}\right)$ and on the zenith angle $(\cos \theta<0.1)$. It contains 5516 neutrino candidates, with a predicted atmospheric neutrino purity of $\sim 90 \%$. The following searches have been performed:

1. a time-integrated full-sky search looking for an excess of events over the atmospheric background in the declination range $\left[-90^{\circ}, 48^{\circ}\right]$. The most significant cluster of events is located at $(\alpha, \delta)=\left(313.20^{\circ}, 64.90^{\circ}\right)$ with a post-trial p-value of $2.7 \%$ (significance of $2.2 \sigma$ ), compatible with background hypothesis;

2. a candidate-list search looking for events in the directions of a predefined list of 50 candidate sources of interest which are known gamma-ray emitters and potential sites for hadronic acceleration. The best candidate source is HESS J0632+057 with a post-trial p-value of $6.1 \%$ (significance of $1.9 \sigma$ ).

In both searches no significant excess over the background has been found and upper limits have been derived. Fig. 1 shows the upper limits derived. The obtained limits are the most stringent ones for a large part of the Southern Sky for sources in the TeV-PeV energy range. IceCube limits in that region of the sky are indeed relevant only above $\sim 1 \mathrm{PeV}$, where the downgoing muon background is effectively reduced. 


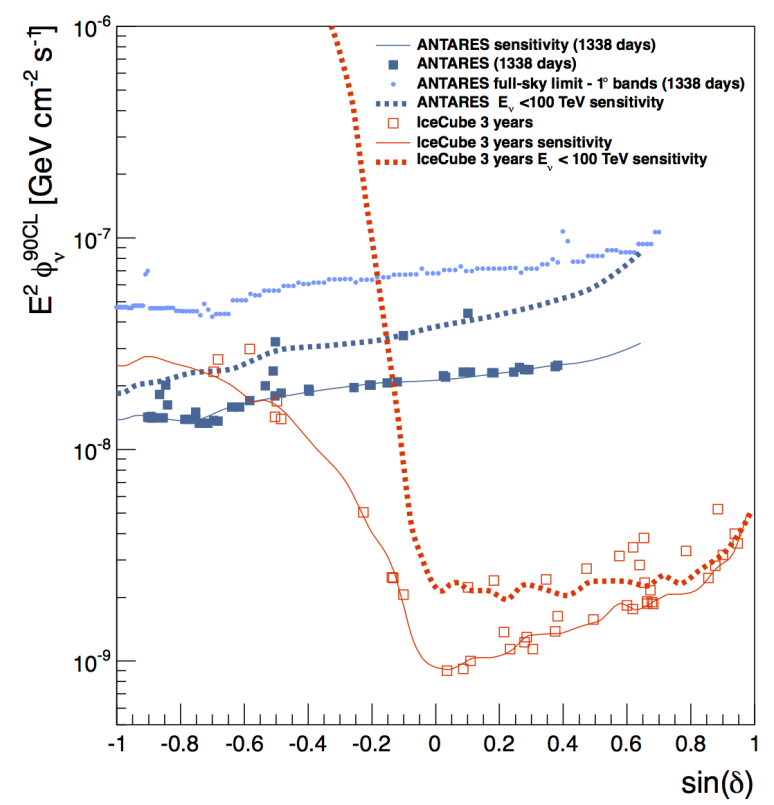

Figure 1: $90 \%$ C.L. flux upper limits and sensitivities for a point-source with an $E_{v}^{-2}$ spectrum as a function of the declination, for six years of ANTARES data. IceCube results are also shown for comparison [5]. The dashed dark blue (red) line indicates the ANTARES (IceCube) sensitivity for neutrino energies lower than $100 \mathrm{TeV}$, showing that the IceCube sensitivity for sources in the Southern Hemisphere is mostly due to events of higher energy (taken from [4]).

\section{Search for cosmic neutrinos from above the horizon}

Until now the point-like sources search has been restricted to the Southern Hemisphere: only up-going events have been studied, in order to reject the background of atmospheric muons produced in cosmic-ray-induced showers. The analysis of down-going events, i.e. the events coming from above the ANTARES horizon, is going on. A big challenge in this analysis is offered by the atmospheric muons which can penetrate through several kilometres of water and reach the detector, thus increasing the background by several orders of magnitude. To retain sensitivity to a neutrino signal flux, it is thus necessary to boost the rejection power. This can be achieved by using a good energy estimator and a valid signal/background separation technique. The most important variable is therefore the energy of the event, but also its coming direction and the track reconstruction quality $(\Lambda)$ are important. The best signal/background separation can be obtained by looking for high energy, mostly horizontal (zenith $\sim 90^{\circ}$ ) and good reconstructed events as shown in Fig. 2.

\section{Summary}

The ANTARES neutrino telescope is in its $8^{\text {th }}$ year of operation. Thanks to its location and to the excellent angular resolution it is yielding the best limits in the world for many (galactic) sources in the Southern Hemisphere, especially for $E_{v}<100 \mathrm{TeV}$. It is expected that by including in the analysis down-going events, by defining zenith-dependent selection criteria, it will be possible to enlarge the detector sensitivity above $40^{\circ}$ in declination. Moreover it will be possible to cover 


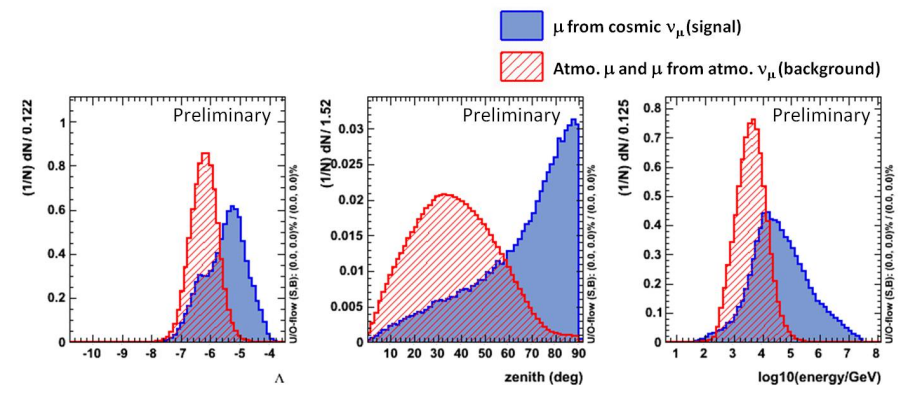

Figure 2: Distribution of $\Lambda$, zenith angle and energy for Montecarlo-generated down-going events in ANTARES, comparing expected signal and atmospheric background.

the full range of accessible particle energies, from the $\mathrm{TeV}$ regime for up-going events to the $\mathrm{EeV}$ regime for the down-going ones, increasing the amount of collected information from possible cosmic ray sources.

\section{References}

[1] M. Ageron et al., "ANTARES: the first undersea neutrino telescope”, Nucl. Instrum. Meth. A $\mathbf{6 5 6}$ (2011) 11 [arXiv:1104.1607 [astro-ph.IM]].

[2] S. Adrian-Martinez, M. Ageron, J. A. Aguilar, I. Al Samarai, A. Albert, M. Andre, M. Anghinolfi and G. Anton et al., "The Positioning System of the ANTARES Neutrino Telescope", JINST 7 (2012) T08002 [arXiv:1202.3894 [astro-ph.IM]].

[3] J. A. Aguilar et al., "Time Calibration of the ANTARES Neutrino Telescope", Astropart. Phys. 34 (2011) 539 [arXiv:1012.2204 [astro-ph.IM]].

[4] S. Adrian-Martinez et al., "Searches for Point-like and extended neutrino sources close to the Galactic Centre using the ANTARES neutrino Telescope", Astrophys. J. 786 (2014) L5 [arXiv:1402.6182 [hep-ex]].

[5] M. G. Aartsen et al., "Search for Time-independent Neutrino Emission from Astrophysical Sources with 3 yr of IceCube Data", Astrophys. J. 779 (2013) 132 [arXiv:1307.6669 [astro-ph.HE]]. 